\title{
TraCKIng SySTEM FOR MOVING OBJECT WITH ForeCASTING
}

\author{
Alexander Kobzev, Natalia Novikova \& Anastasia Lekareva
}
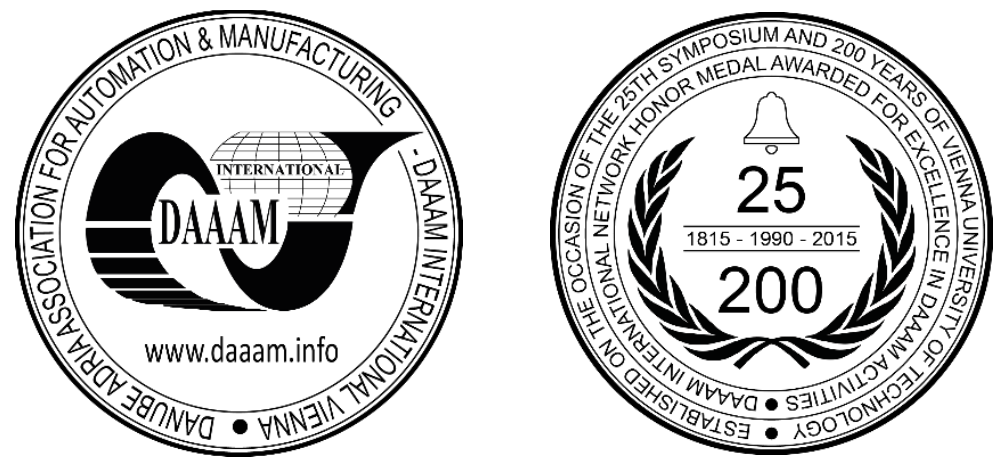

This Publication has to be referred as: Kobzev, A[lexander]; Novikova, N[atalia] \& Lekareva, A[nastasia] (2017). Tracking System for Moving Object with Forecasting, Proceedings of the 28th DAAAM International Symposium, pp.0902-0910, B. Katalinic (Ed.), Published by DAAAM International, ISBN 978-3-902734-11-2, ISSN 1726-9679, Vienna, Austria

DOI: $10.2507 / 28$ th.daaam.proceedings. 125

\begin{abstract}
A characteristic feature of tracking systems for movable objects is the change of trajectory according to various laws. Under the uncertainty of these trajectories, the question of ensuring of a given tracking accuracy is topical. One of the possible options for constructing a rational structure of the automatic control system that enhances the control characteristics is the introduction of a parallel model that generates a signal for «additional control». Hence, it is possible to use two structures of a parallel model: 1) a model similar to the original ACS, but with the main feedback on the adjustable coordinate; 2) a model that forms a given («reference») transient process under various external influences. Studies have shown that the introduction of a parallel model having a structure similar to the original ACS, but with a control object completely covered by feedback, allows to stabilize the response characteristics only in a limited range of external influences. ACSs with a parallel model of the second type have higher response performance. Therefore, both in the first and in the second case it is necessary to introduce an element with a variable transmission coefficient into the circuit of "additional control" signal synthesis, which significantly complicates the structure of the automatic control system and the control problem, since its value depends on many factors.

The introduction of the predictive model allows to improve the response characteristics of the system significantly. The results of the ACS simulation with the parallel predictive model indicate that the maximum absolute error of the controlled quantity does not exceed $1 \%$ of the maximum value of the control input.
\end{abstract}

Keywords: Dynamic objects with the incompletely observed regulated coordinate; predictive control; pattern model; correction algorithms of the controlling action; forecasting of the external effects

\section{Introduction}

When designing automatic control systems (ACS) for various dynamic objects, a careful analysis of the features of their design is required. This analysis is dictated, inter alia, by the requirement of optimal use of all available resources (energy, information, computing, etc.) at each operation stage.

An important feature of a large class of systems and processes is the incomplete observability of the controlled coordinate. This means that part of the control object is not covered by feedback, and therefore, with its typical management structure, its dynamic properties are not taken into account. Such situations arise when certain characteristics of an object are either not available to direct observation, or cannot be automatically measured due to the absence of the required sensors or are measured with a long delay. In addition, cases of inability to use instrumentation due to the 
presence of an aggressive environment, shaking, vibration, and also because of the lack of space to accommodate them during the process, should be explicitly noted [1].

The absence of the feedback on the controlled coordinate (i.e. presence of the unaccounted dynamic of the object of the control) results in the significant decrease of the effectiveness of the control, inability to provide required quality characteristics of the control process which includes non-compliance with the requirements of the stability. [2].

In technical terms, the problem under consideration is the most acute for three types of technical systems: 1) tracking objects on mobile vehicles (land, sea, aviation); 2) industrial robots with angular and anthropomorphic kinematics with «long» arms; 3) metalworking machines (turning, milling and other classes of machines).

One of the possible options for designing a rational structure of the ACS that enhances the control characteristics of the class of objects described earlier is the introduction of a parallel predictive model (PPM). The advantage of this approach, which allowed it to develop successfully in the practice of building and operating control systems, is the relative simplicity of the basic feedback generation loop, combined with the high adaptability. The latter makes it possible to control multidimensional objects with a complex structure and multiple connections including non-linearities, to optimize real-time processes within the constraints on control and controlled variables and to take into account the uncertainties in the descriptions of objects and perturbations [3]

Among the most common methods of predictive control special mention should go to: 1) Dynamic matrix control (DMC). Application area: linear open stationary processes. [5]; 2) Model algorithmic control (MAC). MAC is applicable only for the stable processes with an open scheme [6]; 3) Predictive functional control (PFC). PFC algorithm requires real time optimization method [7]; 4) Extended prediction self-adaptive control (EPSAC). It is characterized by simplicity of the formulated law of control. Possible settings are horizon of the prediction, weighting factor and polynomial filter. Though the real time setting of the predictive horizon influences on the structure of the multi-level predictor and control structure. [8]; 5) Extended horizon adaptive control (EHAC). It doesn't require knowledge of the matrix analysis systems and bears the effect of the output disturbances. However, multimetrical regulators based on one-step criteria of the prediction are sensitive to the choice of the delay structure. [9]; 6) Generalized predictive control (GPC). GPC is applicable for the nonminimum phase systems, with the unstable open loop and changing delay time. It can consider both constant and changing future values of the control points. [10]; 7) Neural inverted predictive control [11].

The aim of this work is an adaption of the forecasting control methods to the objects with the incompletely observed regulated coordinate for the increase of their control characteristics.

\section{Automatic control systems with an incompletely observed controlled coordinate}

The concept of distinguishing a class of automatic control systems with an incompletely observed controlled coordinate can be represented as the following sequence of procedures.

- Analysis of the process or mode of operation of the control object.

- Analysis of the media on which the location of the actuator or object control subsystem.

- Analysis of the structure of the original system. Allocation of subsystems.

- Establishment of the structural interconnection of subsystems in the hierarchy and the formation of control signals and perturbations.

- Isolation of links that generate the non-observability of the coordinates of the state space. The definition of zones and time intervals is not complete observability.

- Determination which links of the system are not covered by the main feedback.

- Designing a model of links not covered by the main feedback [14].

The block diagram of a tracking automatic control system with an incompletely observed controlled coordinate is shown in Fig. 1.

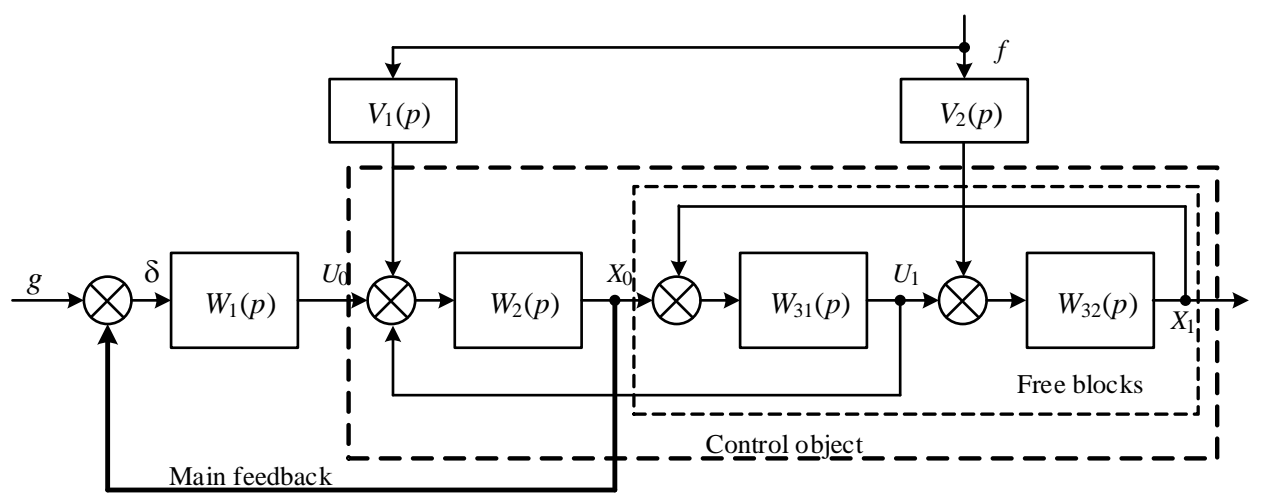

Fig.1. Block diagram of a tracking ACS with an incompletely observed controlled coordinate 
Three blocks of the forward contour are marked on the figure 1: an actuator with kinematic transmission, described by the transfer functions $W_{1}(p), W_{2}(p)$; the control object, partially covered by the main feedback, with the transfer function $W_{31}(p)$; the control object, partially not covered by the main feedback, with the transfer function $W_{32}(p)$. The block diagram also denotes: $V_{1}(p), V_{2}(p)$ - transfer functions of the actuator for perturbation for the covered and not covered parts of the object by the main feedback; $g, f$ - control signal and perturbations; $X_{1}, X_{0}$ - directly adjustable coordinate and its intermediate value, covered by the main feedback of the ACS; $\delta$ - is the error of the system.

Consider the features of the ACS for control of the tracking objects located on mobile vehicles:

- the ACS represents two sequentially linked (i.e. in series) interconnected systems. The first (mobile object) forms perturbations, which are subject to mitigation and stabilization. Coordinate oscillations of the hull are generating control signals and perturbations for the second system that performs direct tracking of mobile objects;

- the law governing the change in the control effect is random and represents a subharmonic function;

- the explicit control signal for the system is not synthesized;

- the signal of the direct path of the system is the error signal obtained from the gyro sensor.

- «platform» represents a beam, fixed in the hinge, with one arm $l_{1}$ at least an order of magnitude shorter than the other $l_{2}$;

- an adjustable coordinate determining the «quality of the technological process» is the extreme point of the longitudinal axis of the long arm beam $l_{2}$;

- the gyroscopic main feedback sensor is located on the short arm of the beam in the immediate vicinity of the hinge axis;

- if the beam is considered to be a nonrigid link, the main feedback sensor does not provide reliable information about the adjustable coordinate under perturbations with large accelerations and with a considerable mass of the beam (platform);

- for constructive and «technological» factors it is impossible to install a gyroscopic feedback sensor at the end of a long arm of a beam (platform).

\section{The concept of predictive control}

Predictive control refers to a class of control algorithms that use models to predict future system reactions. Fig. 2 shows a typical block diagram of the ACS with predictive control.

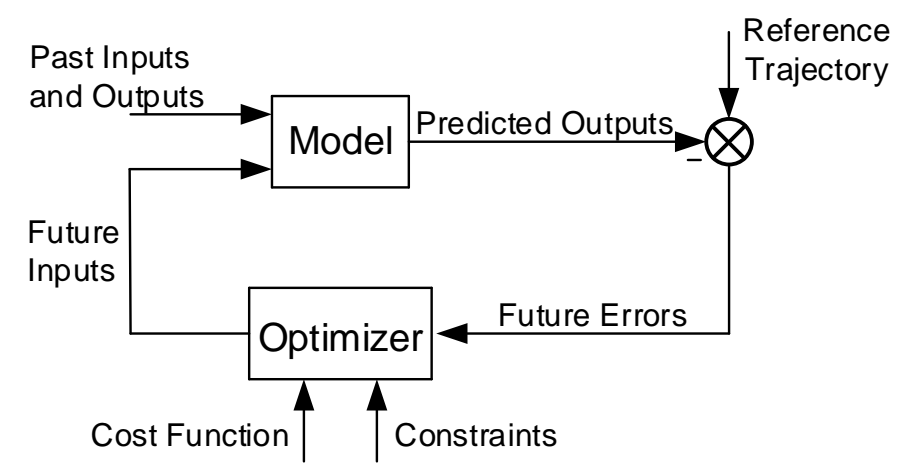

Fig. 2. Block diagram of ACS with predictive control

The implementation of the control with the predictive model can be described as the sequential execution of the following actions [3]:

1. Estimation of the state vector of the control object:

$$
\begin{aligned}
& \hat{x}[k+1]=\hat{A} \hat{x}[k]+\hat{B} u[k]+\hat{H} d[k], \quad \hat{x}[0]=\hat{x}_{0}, \\
& \hat{y}[k]=\hat{C} \hat{x}[k]+w[k],
\end{aligned}
$$

where, $k=0,1,2, \ldots$ - is the clock cycle counter that determines the discrete time moment $t_{k}=k T, \Delta t-$ is the discretization step, $\hat{x}[k] \in E^{n}-$ is the state vector of the control object at time $t_{k}, \hat{u}[k] \in E^{m}-$ is the control vector at time $t_{k}$, and $\hat{y}[k] \in E^{r}$ - is the measurement vector, $d[k] \in E^{n d}$ - external perturbation, $w[k] \in E^{r}$ - noise in the measurements.

2. Selection of control on the forecast (prediction) horizon optimizing the motion of the predictive model (approximating the dynamics of the control object): 


$$
\begin{aligned}
& x[i+1]=f(x[i], u[i]), \quad i=k+j, \quad j=0,1,2, \ldots, \quad x[k]=\hat{x}[k], \\
& y[i]=C x[i],
\end{aligned}
$$

where, $x[i] \in E^{n}$ - is the model state vector, $u[i] \in E^{m}$ - the control vector, $y[i] \in E^{r}$ - the output vector. The predictive model is initialized at the clock cycle $j=0$ by the current state of the real object $\hat{x}[k]$.

As a functional of the control quality of the predictive model, we consider the forecast horizon $P$ in the following form:

$$
J_{k}=J_{k}(y, \Delta u)=\sum_{j=1}^{P}\left[\left(y[k+j]-r[k+j]^{T} R[k+j]\left(y[k+j]-r[k+j]+\Delta u[k+j-1]^{T} Q[k+j] \Delta u[k+j-1]\right],\right.\right.
$$

where, $\quad R[k+j], \quad Q[k+j] \quad$ - given positive-definite matrices, $\quad y=(y[k+j] y[k+j+1] \ldots y[k+P])^{T} \in E^{r P}$, $\Delta \bar{u}=(\Delta u[k+j] \Delta u[k+j+1] \ldots \Delta u[k+P-1])^{T} \in E^{m P}$ - vectors of adjustable and control sequences on the forecast horizon, respectively.

3. Realization of the calculated optimal control for the object on the current clock cycle (from moment $k$ to moment $k+1$ ).

4. Repetition of the steps 1 to 3 for the next cycle, starting from the time moment $k+1$.

The traditional scheme of predictive control has a number of shortcomings that limit its application in real conditions:

- a continuous evaluation of the model state vector is necessary in order to implement the forecast [15], [16]. However, the Wiener or Kalman filters require knowledge of the statistics of the signals, which are not always available. In addition, active uncontrolled perturbations introduce a significant error in the estimation process, and the problem of joint identification of the state vector and perturbations is considered as the problem of mathematical optimisation without a proven global extremum [17];

- for the purpose of designing a robust controller, for example, using optimization $H \bowtie$, one resorts to approximating of the delay element using Pad' series, which leads to inadequate results.

In this article authors propose a modification of the traditional predictive control scheme. In the ACS, a control correction circuit with the predictive model is introduced. The predictive analyzer included in the channel estimates the external influences a step forward, which allows to evaluate the system's responses. In this case, the parallel model generates an additional component of the ACS mismatch signal ahead of time, determined by the extrapolation step. For a model, two types of structures can be considered: 1) a model having a structure similar to the original ACS, but with a control object completely covered by feedback; 2) the model that forms the «reference transient process». In the second case, the structure of the model does not matter, since a system which is built into the parallel system is not the one with a certain scheme and parameters, but an independent structure that forms the required dynamic process.

In this case, we can distinguish three basic structures of the system with a PPM:

1) systems with a parallel predictive management model;

2) systems with a parallel predictable model by perturbation;

3) ACSs for stabilization with a predictive model in the error circuit (here: two predictive models, both for control and for perturbation). Figures $3 \mathrm{a}$ and $\mathrm{b}$ show block diagrams of the ACS with PPMs for control and perturbation.

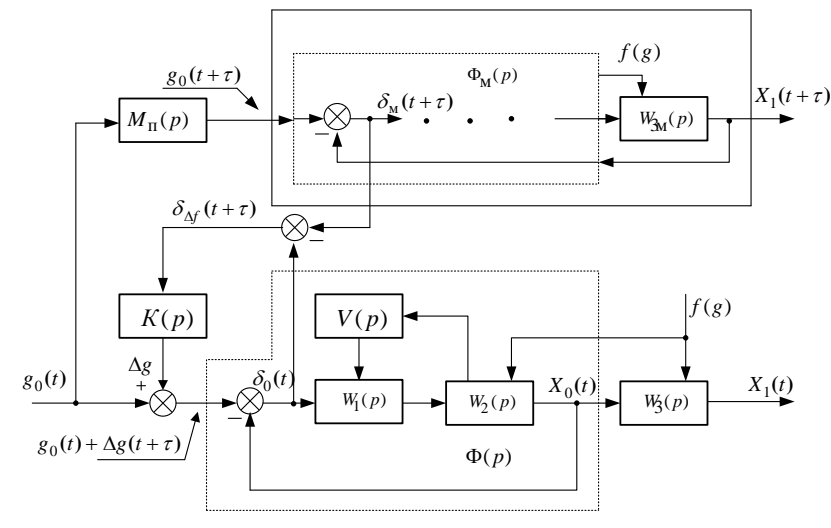

a)

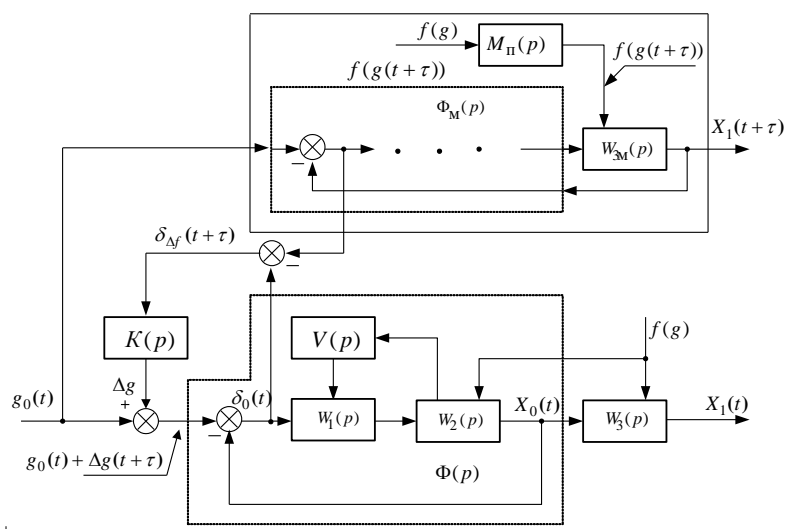

b)

Fig. 3 - block diagrams of ACS with parallel predictive model: a) for control and b) for disturbance 
Features of structures:

1) at the input of the model there is a leading block with the transfer function $M(p)=e^{p \tau}$; where $\tau$ - is the lookahead time;

2) the model of the main circuit includes all links of the direct path, including the transport system; its transfer functions

$$
\Phi_{\mathrm{M}}(p)=\frac{W_{\mathrm{M}}(p)}{1+W_{\mathrm{M}}(p)} \quad \Phi_{f \mathrm{M}}(p)=\frac{V_{1}(p)}{1+W_{\mathrm{M}}(p)}
$$

3) an additional component in the control action is formed on the basis of the difference between the model and system error signals

$$
\delta_{\Delta f}(t+\tau)=\delta_{\Delta f}(t+\tau)-\delta_{0}(t)
$$

4) additional control eventually forms a link with a transfer function $K(p)$.

To synthesize algorithms for additional control, we use the technique proposed in [18]-[21]. Consider the structural diagrams of the formation of an additional component with a forecast in control. There are two possible options: 1) the formation of an autonomous component in the current control signal; 2) the formation of an additional component at the stage of decomposition of the predetermined path in the device for generating control actions.

Thus, depending on the type of control action unit $K(p)$, which is supplied to an error signal should have a transfer function:

1) the first variant of additional management implementation:

$$
\begin{array}{ll}
g(p)=g_{\mathrm{M}}=X=\mathrm{const}: & K(p)=\frac{v_{0}}{p} ; \\
g(p)=\dot{g}_{\mathrm{M}}=\frac{V}{p}, V=\mathrm{const}: & K(p)=\frac{v_{1}}{p^{2}} \\
g(p)=g_{\mathrm{M}}=\frac{\varepsilon}{p^{2}}, \varepsilon=\mathrm{const}: & K(p)=\frac{v_{2}}{p^{3}}
\end{array}
$$

where: $X$ - adjustable coordinate; $V$ - velocity input signal; $\varepsilon$ - acceleration of the reference signal; $v_{0}, v_{1}, v_{2}$ - transmission coefficients in the channel for generating additional control. This method is not related to the methods of control signal synthesis. It can be effectively applied in tracking systems for mobile objects.

2) the second variant of additional control implementation:

$$
\begin{array}{ll}
g(p)=g_{\mathrm{M}}=X=\mathrm{const}: & K(p)=\frac{\gamma_{0}}{p} ; \\
g(p)=\dot{g}_{\mathrm{M}}=\frac{V}{p}, V=\mathrm{const}: & K(p)=\frac{\gamma_{1}}{p} ; \\
g(p)=\ddot{g}_{\mathrm{M}}=\frac{\varepsilon}{p^{2}}, \varepsilon=\mathrm{const}: & K(p)=\frac{\gamma_{2}}{p}
\end{array}
$$

where $\gamma_{0}, \gamma_{1}, \gamma_{2}$ - transmission coefficients in the additional control signal synthesis channel.

\section{The choice of the algorithm for the predictive analyser}

This part of the article is dedicated explicitly to the choice of predictive functions or polynomials and the analysis of the accuracy of their work in real time.

When selecting a method for predicting functions in dynamical systems, it is required to obtain the best approximation within the minimum number of measures. Authors propose an approach to the choice of the algorithm of the predictive model, which includes the following components [14]:

- Analysis of characteristic operation modes of the control object for a reasonable choice of the approximating function. When choosing a predictive function, it is necessary to consider the frequency spectrum of the control signals and perturbations. It is necessary to take into account the own transmission frequency of the servo actuators, the duration 
of the impulse response of the actuator, the precision of tracking and/or stabilization and the frequency properties of the links not covered by the main feedback.

- The choice of the method of calculating of the approximating function. The prediction problem can be solved using interpolation methods, and therefore on this step the problem of «forward interpolation» - extrapolation or approximation of the received data - is formulated.

- Determination of the amount of initial data for prediction with the required accuracy - the number of nodes and their fixation (equidistant or with arbitrary intervals).

- Selection of the extrapolation step based on theoretical data and simulation results, while taking into account the statements above and prediction errors.

The results of the study of the predictive model algorithms are presented in [22]. The analysis of the obtained results testifies to the advantage of the prediction using the Newton interpolation polynomials. High accuracy of calculations with a relatively small computing time, the simplicity of the algorithm makes it most preferable in the construction of automatic control systems with predictive models.

\section{Results of the study}

The investigated movement actuator with the main feedback on its position (DC motor with independent excitation) includes two internal circuits: the voltage and the speed of the motor and the PID controller, and has stable characteristics (no over-adjustment (overshoot) and a specified response rate of $0.2 \mathrm{~s}$ ) which in practice do not depend on external influences. The control object is not covered by the feedback and has a finite rigidity $\mathrm{c}$ and the damping factor $\mathrm{b}$, its design scheme shown in Fig. 4.

The system of differential equations of the dynamics of the control object has the form

$$
\begin{aligned}
& M_{1}-M_{2}=J_{1} \dot{\varphi}_{1} ; \\
& J_{2} \ddot{\varphi}_{2}=c\left(\varphi_{1}-\varphi_{2}\right)+b\left(\dot{\varphi}_{1}-\dot{\varphi}_{2}\right)+M_{n},
\end{aligned}
$$

where $M_{1}, J_{1}$ - dynamic moment and the moment of inertia of the actuator, $M_{2}, J_{2}$ - moment of the elastic forces and reduced moment of inertia of the load, $\varphi_{1}, \varphi_{2}$ - angular displacement between the actuator and the load, $M_{n}$ - external static moment. The moment of the elastic forces $M_{2}$ is a major perturbation and determines the impact not covered by the feedback control of the object during the functioning of ACS. The system of differential equations describing the dynamics of the ACS with an incompletely observable control object is of the sixth order and considers the limitations on the motor current and voltage and nonlinearity of the control object. The ACS research was carried out by simulation of the dynamic modes in the MatLab environment (Simulink). The qualitative parameters of transient processes and the accuracy of adjustment were determined under different laws of the input signal and load parameters (static moment and moment of inertia of the load).

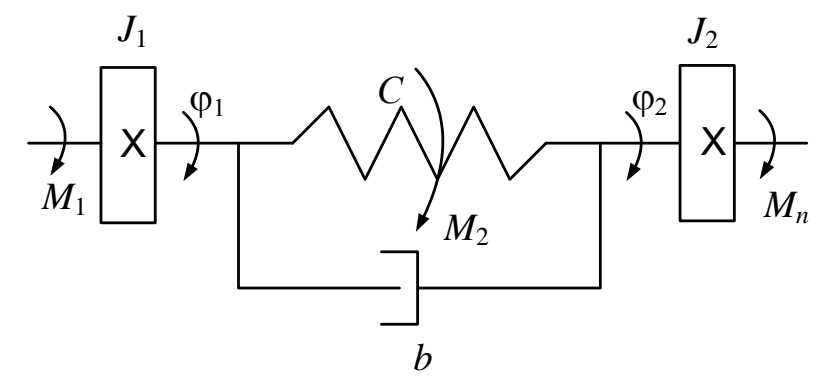

Fig. 4. The design scheme of the control object

The simulation results showed that the lack of complete information on the adjustable coordinate sharply reduces the performance of the ACS, especially with variable external influences. The increase in the control signal causes an increase in the oscillation index in such systems and can lead to instability. An increase in the static moment of the load $M_{\mathrm{H}}$ results in an increase in overshoot during transient conditions and reduce the marginal stability of the system. A similar effect on the dynamics of ACS has a change in load inertia $J_{2}$. With harmonic input signals, increasing their frequency causes a decrease in accuracy, the steady-state dynamic error increases to an unacceptable value.

Studies have shown that the introduction of a parallel model having a structure similar to the original ACS, but with a control object completely covered by feedback, allows to stabilize the dynamic characteristics (eliminate overshoot, shorten the transient process, reduce the error) only under a limited range of external perturbations. With significant changes in the driving force, the model can become a source of additional perturbation. This will often lead to a deterioration in the dynamic characteristics and stability of the ACS.

ACS PPMs of the second type have high dynamic performance. These models tend to include the synthesis of the «additional control» element with variable transmission coefficient $K(g)$ in their circuits. 
The value of this coefficient depends on the value of the reference variable $g$. This inclusion ensures invariance of the ACS to external perturbations and minimize the negative influence of the actuator and load nonlinearities

$$
\Delta g=K(g)\left(\varphi_{2}^{*}-\varphi_{1}\right)
$$

Here $\Delta g$ - «additional control», a difference between the output coordinates of the model - $\varphi_{2}^{*}$ and ACS actuator - $\varphi_{1}$.

Fig. 5 shows the dependency of coefficient of transfer (1) from the magnitude of the rapidly changing reference variable. Results were achieved from simulations under conditions of monotonic transient lasting no more than $0.2 \mathrm{~s}$.

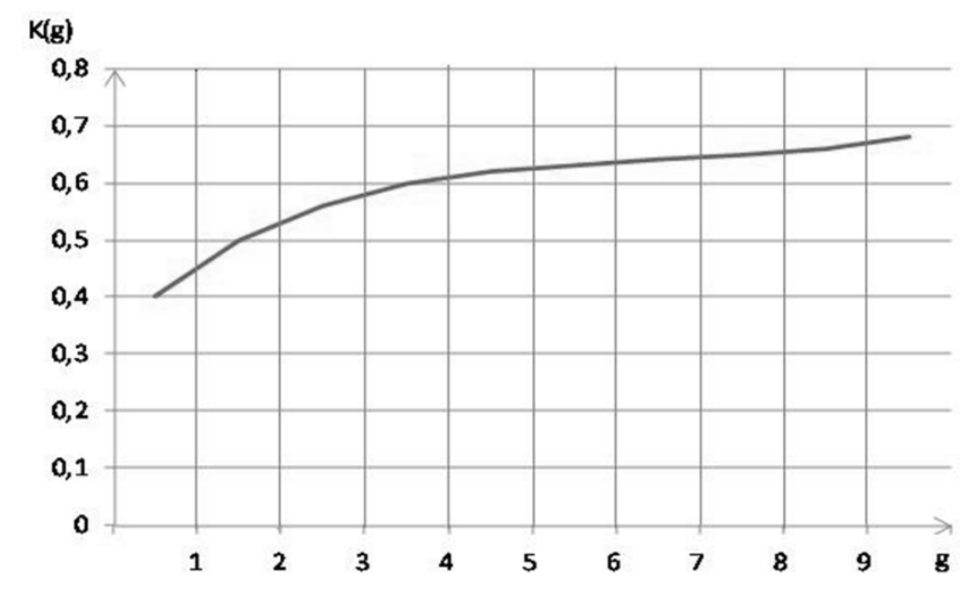

Fig. 5. Dependency of additional transmission coefficient $K(g)$ from the magnitude of the reference variable

A significant influence on the qualitative parameters of the ACS is caused by the nonlinearity of the control object not covered by the main feedback. For example, object dead zone in the control system without a parallel model increases the dynamic error to $20 \%$ of the steady-state value of the output quantity. The introduction of parallel model can significantly reduce the steady-state error, but doesn't completely eliminate the effect of nonlinearity. To ensure the stability of dynamic characteristics in automatic control with a nonlinear control object it is expedient to use an element with a variable transmission coefficient, as in the additional control channel, and in the PPM. Mathematical function of this nonlinear operation correction unit depends on the amount of control. Dependencies of the control channel transmission factors and the model to ensure stable performance of ACS (under conditions of monotonic transient lasting no more than $0.2 \mathrm{~s}$ ) from the magnitude of the rapidly changing input signal are shown in Fig. 6. Similar results are obtained with harmonic input signal with varying amplitude.

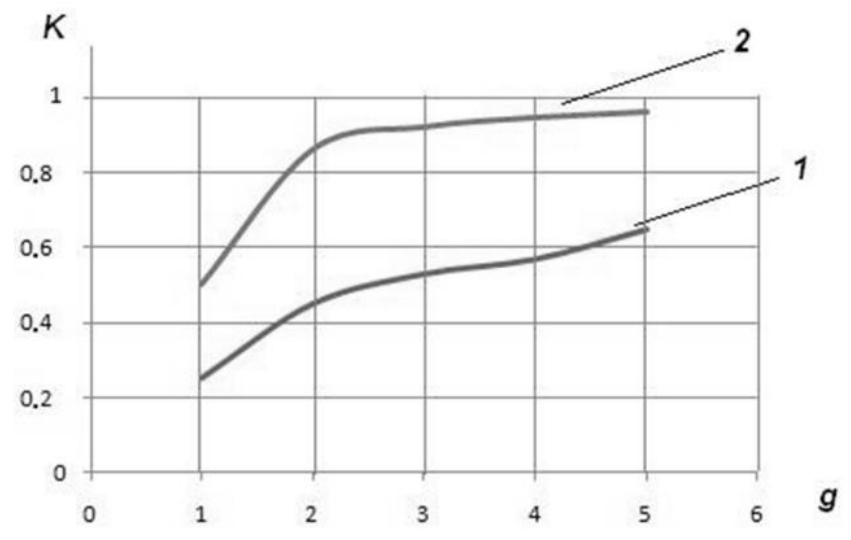

Fig. 6. Dependencies of the transmission coefficients of the nonlinear element in a control channel (1) and parallel model (2) from the magnitude of the input signal

Introduction of the external influence prediction element to the signal synthesis channel of the ACS with PPM allows to improve the dynamic characteristics of the system without resorting to the use of the nonlinear element with a variable transmission ratio, which would significantly complicate the structure of ACS and control problems, because its value depends on many factors.

We investigated the movement modes with different parameters of external influences (static moment and the moment of inertia of the load) using operation algorithm for the predictive element that implements the Newton's second interpolation formula. 
The greatest influence on performance of ACS under harmonic and polyharmonic perturbations is caused by the frequency of their change. Ensuring the stability of ACS characteristics is possible via the variation of prediction time. Fig. 7 shows the dependency of the interpolation step ( $\tau$ - prediction time) from the fundamental frequency $(\omega)$ of external perturbations.

The simulation results demonstrated that the maximum absolute error of the controlled variable does not exceed $1 \%$ of the maximum value of the reference variable.

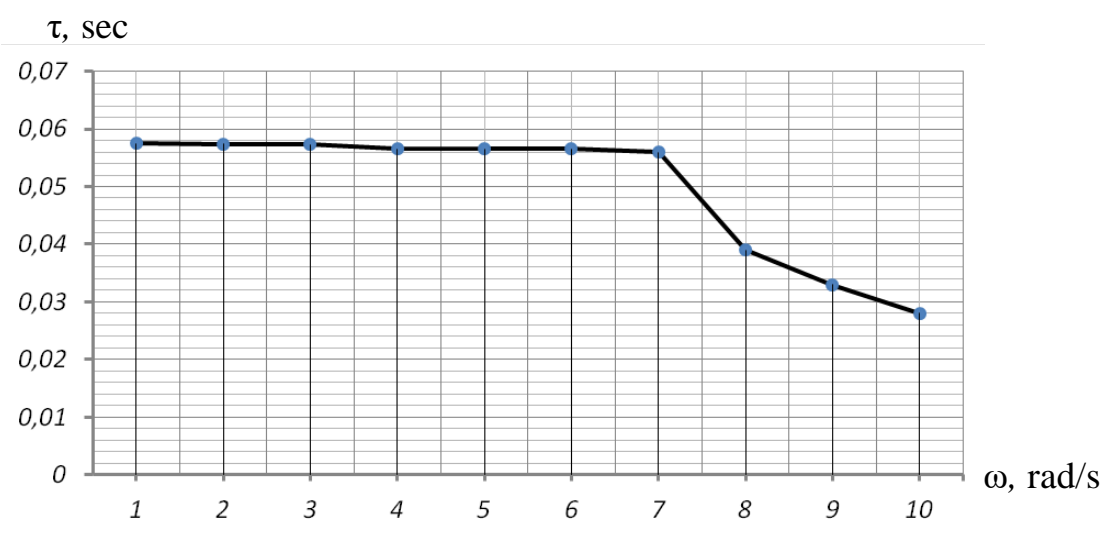

Fig. 7. Dependencies of the prediction time from the input signal

\section{Conclusion}

The absence of the feedback on the controlled coordinate in the objects of the control (i.e. presence of the unaccounted dynamics of the control object) results into the significant decrease of the control effectiveness, inability to provide required quality characteristics of the control process which includes noncompliance of the requirements of the stability. The introduction of the circuit into ACS forming an additional component on the basis of the predictive model is an effective method of the increase of the dynamic characteristics of the objects considered. Herewith it is rational to use the model forming desired transient process in the correction circuit. The algorithm of the work of the predictive analyser is based on the extrapolation Newton's polynom. The results of the simulation showed that maximum absolute uncertainty of the controlled condition doesn't exceed $1 \%$ from the maximum of the reference signal.

The researches made with the introduction of the parallel model without using predictive analyzer showed that for the provision of the desired dynamic characteristics it is necessary to add a link with variable transition factor into the circuit of the formation additional control, and that significantly perplex the structure of the system and control problems.

It is possible to move the development of the control predictive method suggested on the other dynamic objects. It is necessary to make a research in choosing an appropriate time of prediction and synthesis of the algorithms of its determination.

\section{Acknowledgments}

Research is executed with financial support of the Russian Fund of basic researches in the scientific project No. 16-38-00638 mol_a.

\section{References}

[1] Gudzenko, L.I. (1970). Analysis of an unregulated object using the method «black box without entry», In: the collection Identification and equipment for statistical research, Ovsepian F.A. (Ed.), Publishing house Science, Moscow, Russia.

[2] Kobzev A.A.; Mishulin Yu.E.; Novikova, N.A. \& Noskov, E.V. (2010). Analysis of automatic control systems with an incompletely observed controlled coordinate. Proceedings of the Institute of Engineering Physics, Vol. 4, No. 18 (december, 2010), pp. 16-19, ISSN: 2073-8110.

[3] Veremey, E.I.; Eremeev, V.V. (2004). Introduction to management problems based on predictions, Proceedings of the All-Russian Scientific Conference «Design of Scientific and Engineering Applications in the MATLAB Environment», may 25-26, 2004, Moscow, Russia, ISBN 5-201-14971-5200-4, Prangishvili, I.V. (Ed.), pp. 98-115, Publisher house of Institute of Management Problems, Moscow, Russia.

[4] Holkar, K.S. (2010). An Overview of Model Predictive Control. International Journal of Control and Automation, Vol. 3, No. 4, 2010, pp. 47-63, ISSN: 1598-6446.

[5] Cutler, C.R. \& Ramaker, B. (1980). Dynamic Matrix Control - A computer control Algorithm. Proceedings of the Joint Automatic Control Conference, 1980, San Francisco: American Automatic Control Council, Vol. 3, pp. 5- 13.

[6] Mehra, R.K. \& Eterno, J.S. (1980). Model Algorithmic Control for Electric Power Plants. IEEE, 1980, pp. $280-285$. 
[7] Camacho, E.F. \& Bordons, C. (2007). Model Predictive Control, Springer edition, ISBN: 978-1-85233-694-3, London.

[8] De Keyser, R.M.C.; Van De Velde, PH.G.A. \& Dumortier, F.A.G. (1988). A comparative Study of Self-adaptive Long range Predictive Control Methods. Automatica, Vol. 24 (Issue 2), pp. 149-163, ISSN: 0005-1098.

[9] Golden, M.P.; Liu, L.K. \& Ydstie, B.E. (2005). Multivariable Control using Extended Prediction Horizons, Proceedings of 25th Conference on Decision \& Control, December, 1986, Athens, Greece, pp. 1550-1551.

[10] Clarke, D.W. (1987). Generalized Predictive Control: A Robust Self-Tuning Algorithm, Proceedings of American Control Conference, June 10-12, 1987, Minneapolis, Minnesota, pp. 990-995.

[11] Markov, N.A. (2012). Predictive inverse neurocontrol by experimental aerodynamic plant. Vestnik Engineering School of the far Eastern Federal University, Vol. 4 (13), pp. 58-63.

[12] Antos, J[an] \& Kubalcik, M[arek] (2016). Alternative Approach to Optimization in Model Predictive Control Using Hill Climbing Algorithm, Proceedings of the 26th DAAAM International Symposium, B. Katalinic (Ed.), pp. 0856-0864, Published by DAAAM International, ISBN 978-3-902734-07-5, ISSN 1726-9679, Vienna, Austria, DOI:10.2507/26th.daaam.proceedings.119.

[13] Macku, L[ubomir] \& Samek, D[avid] (2016). Self - Organizing Migrating Algorithm in Model Predictive Control: Case study on Semi-Batch Chemical Reactor, Proceedings of the 26th DAAAM International Symposium, B. Katalinic (Ed.), pp. 0238-0246, Published by DAAAM International, ISBN 978-3-902734-07-5, ISSN 1726-9679, Vienna, Austria, DOI:10.2507/26th.daaam.proceedings.033.

[14] Kobzev, A.A.; Mishulin, Yu.E.; Novikova, N.A. \& Lekareva, A.V. (2014). Automatic control systems with parallel predictive model: monograph. ISBN: 978-5-9984-0507-5, Publishing house of the Vladimir State University named after A.G. and N.G. Stoletovykh, ISBN: 978-5-9984-0507-5, Vladimir, Russia.

[15] Liu, Ch.-Sh.; Peng, H. (2002). Inverse-dynamics based state and disturbance observers for linear time-invariant systems. J. of Dynamic Systems, Measurement, and Control, Vol. 124 (July 23, 2002), pp. 375-381, DOI: $10.1115 / 1.1485748$.

[16] Yan, J. \& Bitmead, R.R. (2005). Incorporating state estimation into model predicative control and its application to network traffic control. Automatica, Vol. 41, pp. 595-604, ISSN: 0005-1098.

[17] Camacho, E.F. \& Bordons, C. (2007). Model Predictive Control, Springer edition, ISBN: 978-1-85233-694-3, London.

[18] Novoselov, B.V.; Gorokhov, Yu.S.; Kobzev, A.A. \& Shchitov A.I. (1975). Automatic machines-adjusters tracking systems, Publishing house Energy, Moscow, Russia.

[19] Kobzev, A.A. (1991). Adaptation of the control action in the drives of the assembly robot. News of Higher Educational Establishments. Electromechanics, No. 12, pp. 73-79, ISSN: 0136-3360.

[20] Kobzev, A.A.; Novikova, N.A. \& Lekareva, A.V. (2015). Investigation of adaptation control algorithms for drives of robotic systems using the intercoordinate displacement simulator. News of Higher Educational Establishments. Electromechanics, No. 3, pp. 50-55, ISSN: 0136-3360.

[21] Lekareva, A.V. (2016). Ensuring the invariance of error by perturbation in systems of automatic control of trajectory movements of technological objects. Scientific and Technical Herald of Information Technologies, Mechanics and Optics, Vol. 16, No 5, pp. 787-795, DOI: 10.17586/2226-1494-2016-16-5-787-795.

[22] Kobzev, A.A., Novikova, N.A. \& Lekareva, A.V. (2017). Algorithm selection for the predictive model in control systems with incompletely observable control coordinate. Procedia Computer Science, Vol. 103, pp. 426-431, Available from: http://dx.doi.org/10.1016/j.procs.2017.01.009, Accessed: 2017-08-30. 